\title{
Evaluation of the diagnostic performance of the simple method of computed tomography in the assessment of patients with shoulder instability: a prospective cohort study
}

Tingting Liu' ${ }^{1 \dagger}$, Jianpeng $\mathrm{Ma}^{2 \dagger}$, Hetao $\mathrm{CaO}^{1}$, Dongmei $\mathrm{Hou}^{1}$ and Lin $\mathrm{Xu}^{3 *}$ (i)

\begin{abstract}
Background: Physical examinations may reveal the instability of a glenohumeral joint but cannot diagnose the bony Bankart lesions. Soft tissue Bankart lesion cannot be visualized on traditional radiogram. Magnetic resonance images have high cost and availability issues. The purpose of the study was to access the diagnostic performance of the Computed Tomography (CT) in the assessment of patients with shoulder instability and to diagnose the Bankart and bony Bankart lesions.

Methods: A total of 145 patients with shoulder instability were included in the study. Patients were subjected to clinical examination tests, traditional radiography, and CT. Two orthopedic surgeons, two engineers (trained in musculoskeletal imaging), and two physiotherapists have analyzed the radiological images, CT scans, and the clinical examination tests respectively. The Chi-square test or one-way ANOVA/ Dunnett Multiple comparisons test was performed at $99 \%$ of confidence level.

Results: Sensitivity $(0.972 \pm 0.18$ vs. $1, p=0.11)$ and accuracy $(0.942 \pm 0.17$ vs. $1, p<0.0001, q=3.88)$ for the clinical examination tests combining the traditional radiological images were same to CT. However, the clinical examination tests combining the traditional radiological images had more inconclusive results (5 vs. 1), false-positive results (6 vs. 5), and false negative results (4 vs. 1) than CT. The area that detects the Bankart and bony Bankart lesions at least one time for CT was higher than that of the clinical examination tests combining the traditional radiological images.
\end{abstract}

Conclusion: $C T$ should be considered for evaluation in patients with shoulder instability and suspected Bankart and bony Bankart lesions.

Trial registration: Researchregistry3990 dated 15 December 2014 (www.researchregistry.com).

Keywords: Bankart lesions, Clinical examination tests, Computed tomography, Traditional radiological images, Twin trial

\footnotetext{
* Correspondence: LivelyLenopalin@yahoo.com

${ }^{\dagger}$ Tingting Liu and Jianpeng Ma contributed equally to this work.

${ }^{3}$ Department of Radiology, PLA general hospital, No.28 Fuxing Road, Haidian

District, Beijing 100000, China

Full list of author information is available at the end of the article
}

(c) The Author(s). 2018 Open Access This article is distributed under the terms of the Creative Commons Attribution 4.0 International License (http://creativecommons.org/licenses/by/4.0/), which permits unrestricted use, distribution, and reproduction in any medium, provided you give appropriate credit to the original author(s) and the source, provide a link to the Creative Commons license, and indicate if changes were made. The Creative Commons Public Domain Dedication waiver (http://creativecommons.org/publicdomain/zero/1.0/) applies to the data made available in this article, unless otherwise stated. 


\section{Background}

A glenohumeral joint is most frequently dislocated [1]. The recurrent shoulder instability affected patients can develop significant functional deficiencies and symptoms of chronic instability [2]. The reason behind recurrent instability is a glenoid bone loss or the Bankart lesion. Anterior shoulder dislocation due to structural damage to the anterior-inferior labrum with tearing of the anterior capsule is called the Bankart lesion. When it includes osseous fragments, it is called the bony Bankart lesion. The treatment involved in the reattachment of the lesion is arthroscopy with surgical suture [3]. The reason behind the Bankart lesion is a loss of $25 \%$ and/or more of the width of the inferior glenoid, which leads to a loss of bony support [4]. The glenoid bone loss is frequent in shoulders with the Bankart lesion [5].

The complexity of the combined motions of the degree of the scapulothoracic and the glenohumeral joints creates difficulties in preoperative quantification and identification of the Bankart and bony Bankart lesions for decision making of arthroscopy or open procedures [6]. Surgeons may fail in the surgical repair of the Bankart and bony Bankart lesions if not follow protocol for workup and have addressed a bony problem without being aware of it by the differential diagnosis. Moreover, the management of the Bankart and bony Bankart lesions after a failed surgery could be challenging. Therefore, an adaptation of proper diagnostic modality is crucial in the detection of the Bankart and bony Bankart lesions [7]. The optimal treatment for the Bankart and bony Bankart lesions remains controversial [8]. The accepted diagnostic method of quantifying the Bankart and bony Bankart lesions is not available [9] because physical examinations may reveal instability of the glenohumeral joints but cannot diagnose the Bankart or bony Bankart lesions. Generally, orthopedic surgeons have preferred radiography, the Computed Tomography $(\mathrm{CT})$, and magnetic resonance imaging (MRI) in the decision making of surgeries [6].

Soft tissue Bankart lesion cannot be visualized on traditional radiogram. With a Grashey view, an external and internal rotation view, an axillary lateral view, a scapular-Y view, a West Point view, a Stryker notch view, and an apical oblique (Garth) view, the sensitivity of traditional radiography for detection of significant glenoid bone loss is not more than 50\% [2]. Moreover, an acute fracture of the glenoid rim is often inaccurately quantified by traditional radiography [10]. Therefore, traditional radiography is underestimated bone defects. MRI has high cost and availability issues [3]. MR images are suggested only if the traditional radiography will fail to provide the necessary information for decision making of surgeries [11]. Therefore, there is a strong need for a consensus on the universally accepted diagnostic method of quantifying the Bankart and bony Bankart lesions.
The goal of the study was to access the diagnostic performance of the simple method of CT in the assessment of patients with shoulder instability and to diagnose the Bankart and bony Bankart lesions. The secondary endpoint of the study was to compare sensitivity and accuracy of the clinical examination tests combining the traditional radiological images with CT for decision making of arthroscopy or open procedures for repair of the bony problem of shoul$\operatorname{der}(\mathrm{s})$ at level 2 of evidence (Table 1) without conflict of interest.

\section{Methods}

The study had adhered 2013 Declarations of Helsinki, standards for reporting of diagnostic accuracy studies (STARD) guidelines, and the law of China. The work has been reported in line with the strengthening the reporting of cohort studies in surgery (STROCSS) criteria [12].

\section{Inclusion criteria}

Patients age 18 years and above, with a complaint of progressively reduced shoulder function, pain located at the front and lateral side of the shoulder, load-dependent pain, or/and delocalization of the shoulder available with outpatient setting of the PLA general hospital, China, Affiliated Hospital of Nantong University, China, and Dingbian County People's Hospital, China from 18 December 2014 to 1 February 2018 were included in the study. Patients who had a bony problem of shoulder(s) and signed an informed consent form were subjected to a pre-index test (the pre-index tests were performed for eligible participants screening purposes).

\section{Pre-index tests}

Functional outcomes were measured in pre-index tests as follows to confirm glenoid bone loss:

\section{Oxford instability shoulder score}

It contains 12 items questionnaires with four responses $(0-3)$. The total score (the sum of 12 items) ranged from 0 (excellent) to 48 (the worst) [13].

Table 1 Level of Evidence

\begin{tabular}{ll}
\hline Level & Diagnostic study \\
\hline 1 & Testing previously developed diagnostic criteria \\
2 & Testing diagnostic criteria with gold standard \\
3 & Review article/Meta-analysis on STARD studies \\
4 & Case study \\
5 & Expert opinion \\
\hline
\end{tabular}

STARD Standards for reporting of diagnostic accuracy studies 


\section{Western Ontario shoulder instability index}

It has 21 items questionnaires on a visual analog scale score. The total score (the sum of 21 items) ranged from 0 (the excellent) to 100 (the worst) [13].

\section{Simple shoulder test score}

It consists of 12 questions with dichotomous response options, which are scored 0 or 1 . The total score (the sum of 12 items) ranged from 0 (the worst) to 12 (the excellent) [13].

\section{Disability of the arm, shoulder, and hand score}

It is a measurement of physical function and symptoms in patients with musculoskeletal disorders from any condition in any joint in the upper extremity. It has 30-questions (grading 0-3). The average of item scores, subtracting one, and multiplying the result by 25 is the resulting score. It has the range from 0 (no disability) to 100 (extreme disability) [14].

\section{Exclusion criteria}

Patients age younger than 18 years and not signed informed consent form were excluded from the study. Patients who had frozen shoulder and arthritis were excluded from the study. Patients who had oxford instability shoulder score, four and less than four were excluded from the study.

A total of 145 patients with the proved shoulder instability were enrolled in a prospective cohort study. The demographic parameters of the enrolled patients are presented in Table 2. STARD flow diagram of the study is presented in Fig. 1.

\section{Diagnosis of the Bankart and bony Bankart lesions Clinical examination tests}

Physical examinations of shoulders were performed as follows:

\section{Apprehension test}

The enrolled patients had kept in supine position with the arm in $90^{\circ}$ abductions, the elbow in $90^{\circ}$ flexions, and maximum external rotation. The evaluator had applied an anterior, external, rotatory force [15].

\section{The relocation test}

In apprehension test position, the force was applied to the humeral head in the posterior direction [16].

\section{The anterior release/ the surprise test}

The patient's affected arm was maintained in the position of apprehension and the pressure on the humeral head was suddenly released by the evaluator [17].

\section{The anterior drawer test}

Patients were kept in supine positions and affected hand was on the examiner's axilla, the arm was held in 80 $120^{\circ}$ of abduction, $0-20^{\circ}$ of forwarding flexion, and 0 $30^{\circ}$ of external rotation. With one hand, the evaluator had stabilized the scapula by applying force on the coracoid process. The other hand was grasped the humeral and drew it out anteriorly [18].

\section{The load and shift test/ the push-pull test}

Patients were kept in set positions. With the dominant hand, the evaluator was grasped the patient's elbow and the non-dominant hand (of the evaluator) was grasped the patient's upper arm. The evaluator was positioned the patient's arm in $90^{\circ}$ of abduction in the scapular plane with no rotation and centered the humeral head of subject on the glenoid by applying a force along the axis of the humeral with the dominant hand to shift the humeral head in the anterior direction [19].

\section{The Hyperabduction test}

Patients were kept in standing positions, the elbow was flexed at $90^{\circ}$, the forearm was horizontal, and the evaluator was standing behind the patients. With the evaluator's forearm of the dominant hand, the shoulder girdle was pushed down firmly, while the evaluator's non-dominant hand was lifted the patient's upper limb, which was relaxed in the abduction [13].

All tests were performed by two physiotherapists (evaluators; three years of experience) who were blinded regarding radiological images and in order of those have written. The interpretation of observations of all the tests for considering them positive or negative are presented in Table 3.

\section{Traditional radiographs}

All enrolled patients were subjected to axillary traditional radiographs (GE Healthcare, Chicago, IL, USA) at an axial position and at $90^{\circ}$. Conventional axillary lateral, anteroposterior (in exo-rotation or in endo-rotation) radiographs and the Grashey view of the affected shoulder were performed (Fig. 2).

\section{CT scans}

Spiral CT (CATIA, France) was performed at $1.25 \mathrm{~mm}$ thickness and $0.625 \mathrm{~mm}$ interval of affected shoulder to confirm or exclude the Bankart and Bony Bankart lesions (Fig. 3).

\section{Image analysis}

Radiological images were checked by a radiologist (three years of experience) under the lightbox (Efftronics Systems Pvt. Ltd., China). A small bone spur in traditional radiological images of the shoulder was considered as the Bankart or bony Bankart lesions present (Fig. 4). The 
Table 2 The demographic parameters of the enrolled patients

\begin{tabular}{|c|c|c|}
\hline Characters & & Populations \\
\hline Patients enrolled in the study (sample size) & & 145 \\
\hline \multirow[t]{2}{*}{ Gender } & Male & $66(46)$ \\
\hline & Female & $79(54)$ \\
\hline Age (years) & & $28.52 \pm 7.56$ \\
\hline \multirow[t]{3}{*}{ Reduced shoulder function } & DS & $114(79)$ \\
\hline & NDS & $29(20)$ \\
\hline & Both & $2(1)$ \\
\hline \multirow[t]{3}{*}{ Pain located at the front side of the shoulder } & DS & $117(81)$ \\
\hline & NDS & $24(17)$ \\
\hline & Both & $4(2)$ \\
\hline \multirow[t]{3}{*}{ Pain located at the lateral side of the shoulder } & DS & $113(78)$ \\
\hline & NDS & $30(21)$ \\
\hline & Both & $2(1)$ \\
\hline \multirow[t]{3}{*}{ Load-dependent pain } & DS & $125(86)$ \\
\hline & NDS & $22(18)$ \\
\hline & Both & $8(6)$ \\
\hline \multirow[t]{3}{*}{ Delocalization of the shoulder } & DS & $112(77)$ \\
\hline & NDS & $33(23)$ \\
\hline & Both & $0(0)$ \\
\hline \multirow[t]{2}{*}{ Incidence of event } & The first time & $133(92)$ \\
\hline & The second or third time & $12(8)$ \\
\hline \multirow[t]{4}{*}{ Functional outcome measures } & Oxford Instability Shoulder Score $(48-0)^{a}$ & $20.15 \pm 1.89$ \\
\hline & Western Ontario Shoulder Instability Index (0-100) ${ }^{a}$ & $44.57 \pm 5.28$ \\
\hline & Simple Shoulder Test score $(0-12)^{a}$ & $8.52 \pm 1.13$ \\
\hline & Disability of the Arm, Shoulder, and Hand score $(100-0)^{a}$ & $25.47 \pm 2.58$ \\
\hline
\end{tabular}

Constant data are considered as number (percentage) and continuous data are presented as mean \pm SD

${ }^{a}$ The range is reflected as most impaired to the least impaired condition

DS: The dominant side

NDS: The non-dominant side

Oxford Instability Shoulder Score: 0: excellent, 48: The worst

Western Ontario Shoulder Instability Index: 0: excellent, 100: The worst

Simple Shoulder Test score: 0: excellent, 12: The worst

Disability of the Arm, Shoulder, and Hand score: 100: No disability, 0: extreme disability

Enrolled patients had more than one type of demographic characters regarding the bony problem in the shoulder(s)

All patients have China PR origin

surgeons had decided the Bankart or bony Bankart lesions and taken the decision of arthroscopy or open procedures. CT scans were evaluated using CATIA (CATIA V5R21; Dassault Systemes, France). The percentage missing area was calculated by surface area missing and the inferior glenoid circle (Fig. 5) as per Eq. 1 (surface area method) [20]. The Bankart and/or bony Bankart lesions was confirmed if the percentage missing area was minimum $20 \%$ [3].

The percentage missing area

$$
=\frac{\text { The eroded missing area }}{\text { The inferior glenoid circle }} \times 100
$$

Surgery curative was considered as 'gold standard' in all the operative case. If any kind of bony problem (e.g. inflammation, rupture, ligament straitening, Hill-Sachs lesion) had found in diagnosis, which was lead to arthroscopy or opens surgical procedure and actually it was the Bankart and/or bony Bankart lesions considered as a 'false negative'. If the Bankart and/or bony Bankart lesions had found in diagnosis, which was lead to surgery and actually it was the other than the bony problem (frozen shoulder, arthritis, Hill-Sachs lesion) considered as a 'false positive'. The accurate Bankart and bony Bankart lesions absent category were also found in all diagnosis because all patients who had 


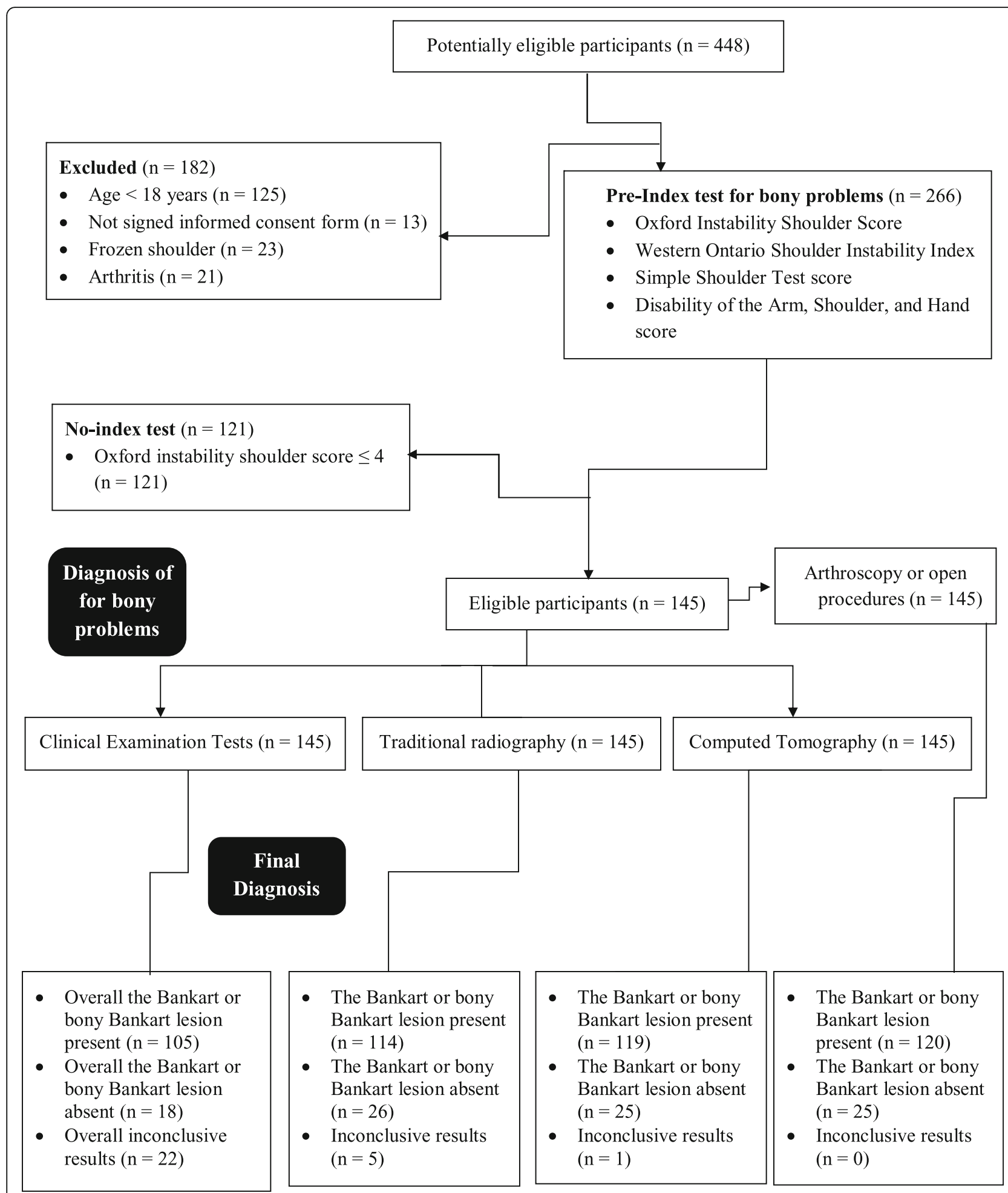

Fig. 1 STARD flow diagram of the study

to complain of the shoulder(s) instability were included and there is no any accurate clinical test until the time to confirm the Bankart and bony Bankart lesions.

\section{Beneficial analysis of diagnostic methods}

Decision-making curve for detection of the Bankart and bony Bankart lesions was drawn by decision curve analysis as per Eqs. 2 and 3 [21]: 
Table 3 Interpretation of clinical examination tests

\begin{tabular}{lll}
\hline Clinical Examination Tests & Observations regarding patients feeling after the test & \\
\cline { 2 - 3 } & Considered positive & Considered negative \\
\hline Apprehension Test & An apprehensive feeling & Only pain noticed \\
The Relocation Test & Apprehensive feeling or pain is reduced & $\begin{array}{l}\text { Apprehensive feeling or pain is not } \\
\text { reduced }\end{array}$ \\
The Anterior Release/ the Surprise Test & An apprehensive experience & Only pain noticed \\
The Anterior Drawer Test & shoulder \\
The Load and Shift Test/ the Push-Pull & displayed apprehension & Only pain noticed \\
Test & Arm hyper-abducted $\geq 105^{\circ}$ & No apprehension \\
The hyperabduction Test & Arm hyper-abducted $<105^{\circ}$
\end{tabular}

Each patient underwent physical examinations total four times by two physiotherapists (blinded regarding radiological images)

Decision making of the Bankart and bony Bankart lesions

$=$ True Positive ratio of the Bankart and bony Bankart lesions

-(False positive ratio of the Bankart and bony Bankart lesions

$\times$ Weighting factor)

Weighting factor $=$

A level of diagnostic confidence above ithad performed arthroscopy or opens surgical procedure

1-A level of diagnostic confidence above it arthroscopy or opens surgical procedure had performed

Weighting factor: The risk of overdiagnosis.

\section{Inter-and intra-observer reliability}

For purposes of the reliability of assessments, two orthopedic surgeons were analyzed twice times the traditional radiological images, two engineers trained in musculoskeletal imaging assessments (this is diploma course for musculoskeletal imaging assessments in PR. China after $\mathrm{MD}$ radiology; three years of experience in image analysis) were evaluated the CT scans twice, and two physiotherapists were evaluated clinical examination tests twice (each patient underwent physical examinations four times). All were blinded to the others' conclusions.

\section{Cost of diagnosis}

Cost of diagnosis was included hospitalization, charges of experts, and radiographers.

\section{Statistical analysis}

Kappa statistics (considering kappa value $(\mathrm{k}) \leq 0.4$ low, $0.41-0.6$ moderate, $0.61-0.8$ substantial, and $\geq 0.81$ perfect inter-and intra-observer reliabilities) were used to determine reliability [22]. One-way analysis of variance (ANOVA) following Dunnett Multiple comparisons tests (considering critical value $(q)>4.148$ as significant) was performed to compare constant diagnostic parameters [23] and the Chi-square test was performed for categorical data. All results were considered significant at 99\% of confidence level. InStat (vWindow, GraphPad, IL, USA) was used for statistical analysis.

\section{Results}

Functional outcome measures were strongly correlated with the Bankart and bony Bankart lesions. $k$ for physiotherapists, orthopedic surgeons, and trained engineers (in musculoskeletal imaging) were greater

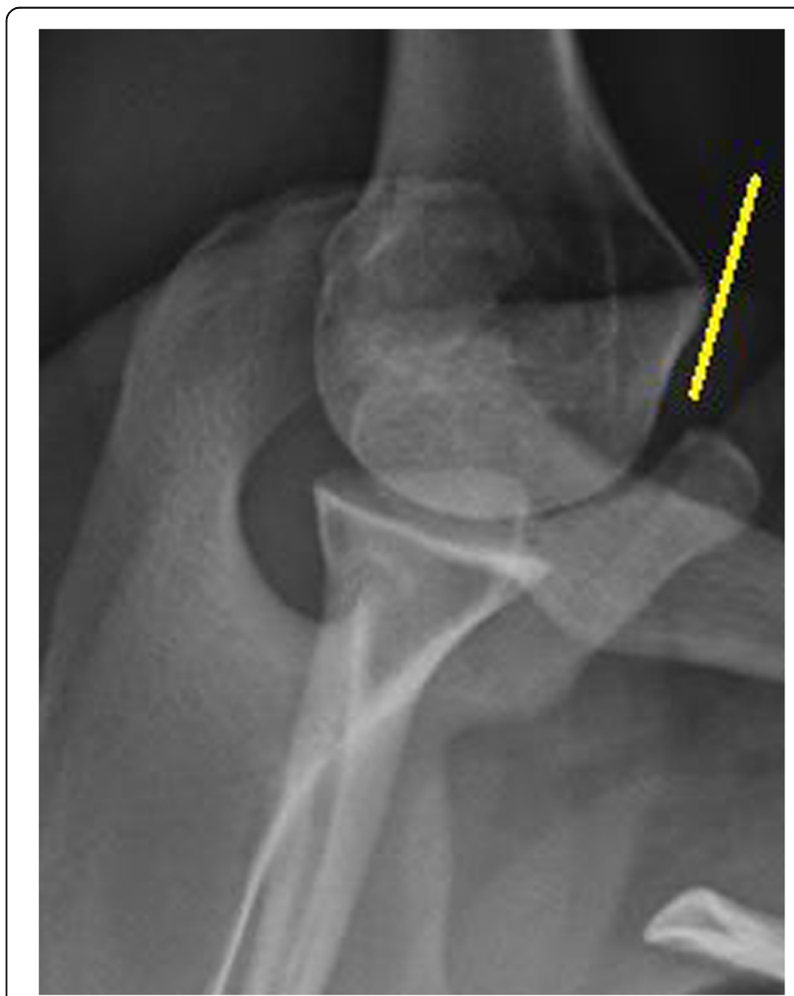

Fig. 2 Traditional radiological image of the shoulder with the Bankart lesions. The yellow line indicates the Bankart lesions. The Grashey view of the patient's shoulder. Images were checked by two radiologists 


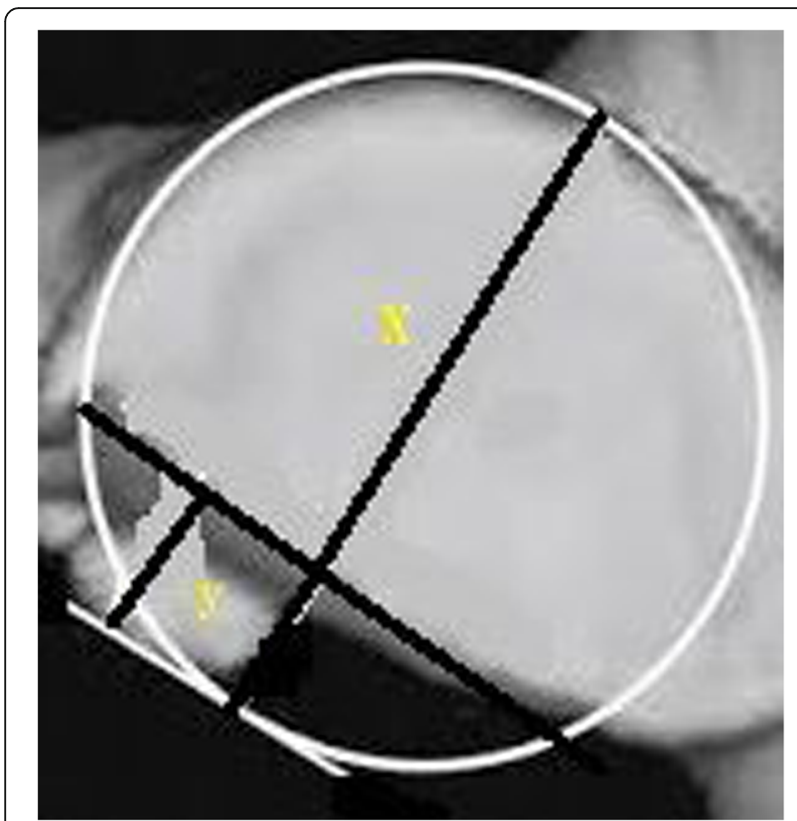

Fig. 3 The Computed Tomography of the patient's shoulder with the Bankart lesions. X: The inferior glenoid circle. Y: The eroded missing area. Images were checked by two engineers (diploma course in musculoskeletal imaging assessments in PR. China after $M D$ radiology) trained in musculoskeletal imaging

than 0.81 indicated perfect inter-and intra-observer reliabilities (Table 4).

Among clinical examination tests, surprise test has high sensitivity $(0.917 \pm 0.15)$ and the anterior drawer test has high accuracy $(0.811 \pm 0.18)$. The area under the curve for the clinical examination tests combining the traditional radiological images was fallen down than CT $(0.76 \pm 0.11$ vs.

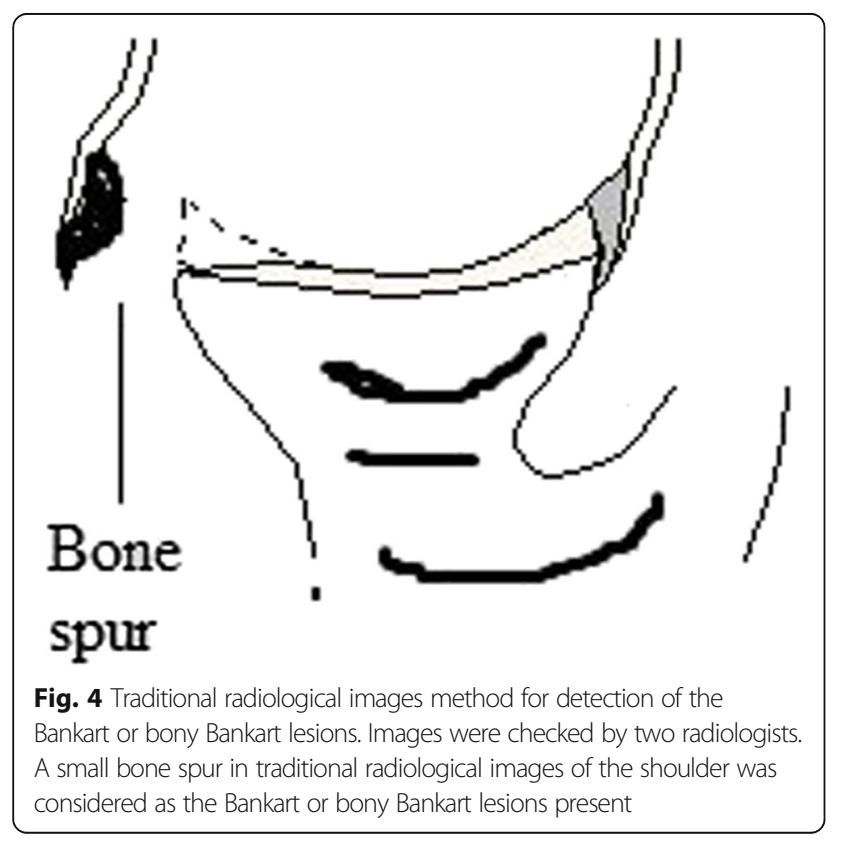

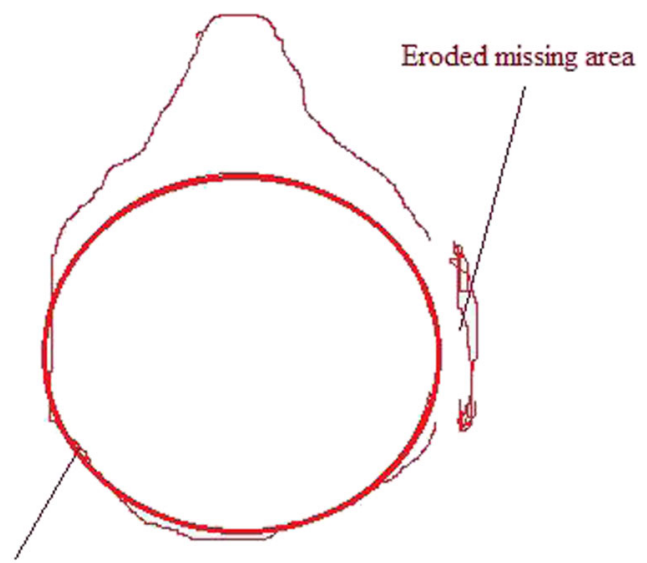

The inferior glanoid circle

Fig. 5 Surface area method of the simple Computed Tomography in the assessment of patients with shoulder instability and to diagnose the Bankart and bony Bankart lesions. Images were checked by two engineers (diploma course in musculoskeletal imaging assessments in PR. China after MD radiology) trained in musculoskeletal imaging. The Bankart and/or bony Bankart lesions was confirmed if the percentage missing area was minimum $20 \%$

$0.89 \pm 0.14, p<0.0001, q=9.39)$. Overall, the clinical examination tests combining the traditional radiological images had provided limited information for decision making of surgeries (Fig. 6). With respect to CT, sensitivity for the clinical examination tests combining the traditional radiological images had no statistical significance difference $(0.972 \pm 0.18$ vs. $1, p=0.11)$ even for accuracy there were no statistical significance difference $(0.942 \pm 0.17$ vs. $1, p<0.0001, q=3.88)$. However, the clinical examination tests combining the traditional radiological images had more inconclusive results (5 vs. 1), false-positive results (6 vs. 5), and false negative results (4 vs. 1) than CT (Table 5). CT was flawless in the decision making of surgeries for the Bankart and bony Bankart lesions than the clinical examination tests combining the traditional radiological images (Table 6).

The area that detects the Bankart and bony Bankart lesions at least one time for $\mathrm{CT}$ was higher than that of clinical examination tests combining the traditional radiological images (Table 7). At 0-50\% level of diagnostic confidence, orthopedic surgeons had preferred the clinical examination tests combining the traditional radiological images but these tests did not give any sufficient information for decision making of surgery. However, above $50 \%$ of the level of diagnostic confidence for the clinical examination tests combining the traditional radiological images, patients had a risk of overdiagnosis and overtreatment. In the range of $0-75 \%$ of the level of diagnostic confidence, CT was the most reliable imaging modality and above $75 \%$ of the level of diagnostic confidence, CT had a risk of overdiagnosis and overtreatment (Fig. 7). 
Table 4 Reliability of assessments

\begin{tabular}{|c|c|c|c|}
\hline Parameters & $\begin{array}{l}\text { Overall Clinical examination } \\
\text { tests }\end{array}$ & $\begin{array}{l}\text { The traditional radiological } \\
\text { images }\end{array}$ & Computed Tomography \\
\hline Sample size & 145 & 145 & 145 \\
\hline Evaluators & Physiotherapists & Orthopedic surgeons & aEngineers trained in musculoskeletal imaging \\
\hline Tested Material & Shoulders of subjects & Traditional radiological images & Computed Tomography images \\
\hline Numbers of evaluators & 2 & 2 & 2 \\
\hline $\begin{array}{l}\text { Numbers of test/images } \\
\text { performed }\end{array}$ & 4 & 1 & 1 \\
\hline Numbers of test/images analyzed & 4 & 2 & 2 \\
\hline Tool for decision making & $1 \times 1$ table & Lightbox & CATIA V5R21 software \\
\hline${ }^{\mathrm{b}}$ The method adopted & Apprehension tests & A small bone spur in images & $\begin{array}{l}\text { The glenoid ratio method (\% missing area } \geq \\
\text { 20\%) }\end{array}$ \\
\hline kappa value & 0.811 & 0.825 & 0.891 \\
\hline
\end{tabular}

kappa value $\leq 0.4$ low; $0.41-0.6$ moderate; $0.61-0.8$ substantial; and $\geq 0.81$ perfect inter-and intra-observer reliabilities

a Diploma course in musculoskeletal imaging assessments in PR. China after MD radiology

${ }^{b}$ for decision making of the Bankart lesion

The costs of clinical examination tests, the traditional radiological images, clinical examination tests combining the traditional radiological images, and CT were $8.59 \pm$ $0.59 \$ /$ patient, $12.64 \pm 1.21$ \$/patient, $21.11 \pm 1.68$ \$/patient, and $47.65 \pm 4.24 \$$ patient respectively. The cost of CT examinations was higher than the clinical examination tests combining the traditional radiological images $(p<0.0001, q=95.05$, Fig. 8).

After surgeries radiographs showed the union of the grafts and no incidents of re-dislocation during the follow-up period (Fig. 9).

\section{Discussion}

In the study, clinical examination tests alone were not provided sufficient information and surgeons were preferred radiological images for decision making of surgeries. There are high varieties of diagnostic modalities available for diagnosis of the instability of shoulders. Among the available methods, apprehension tests are the most frequently used combing traditional radiological images [24]. The Bankart and bony Bankart lesions are difficult to diagnose and there are more chances for failure of surgical procedures [25] because of the low sensitivity of radiological modalities [23]. Moreover, the adoption of the diagnostic method before the surgical procedure for the repair of the bony problem(s) of the shoulder is based on a number of dislocations and prior shoulder trauma [13]. The results of the study were not in line with an available study [13] but in line with the review article [26]. Clinical examination tests combing traditional radiological images

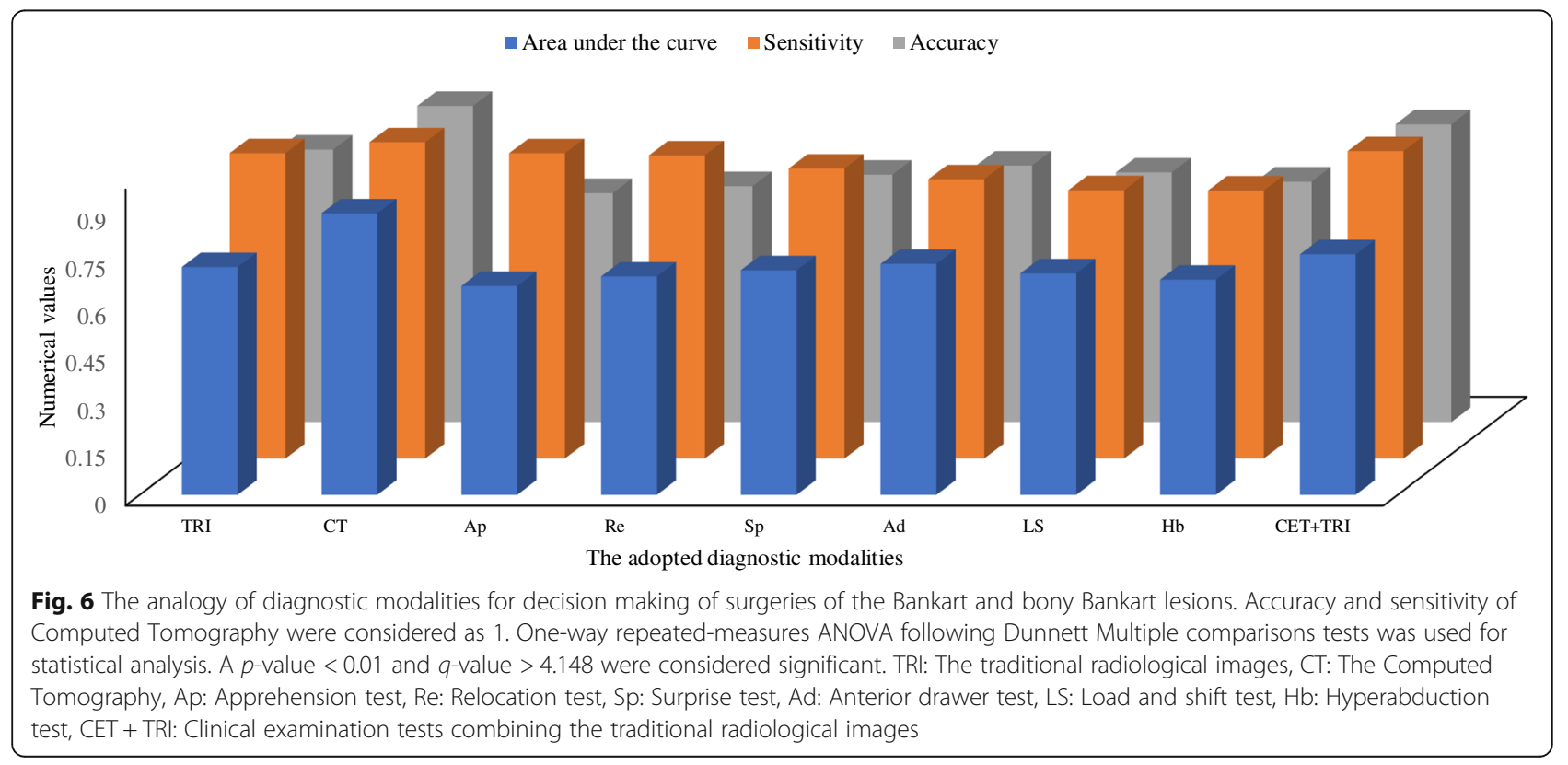


Table 5 Parameters of the adopted diagnostic methods for evaluation of the Bankart and bony Bankart lesions

\begin{tabular}{|c|c|c|c|c|c|c|c|c|c|c|c|}
\hline \multirow[t]{2}{*}{ Parameters } & \multicolumn{6}{|l|}{ CET } & \multirow[t]{2}{*}{ TRI (7) } & \multirow[t]{2}{*}{ CET + TRI (9) } & \multirow[t]{2}{*}{ CT (10) } & \multicolumn{2}{|c|}{ SA 9 vs. 10} \\
\hline & Ap (1) & $\operatorname{Re}(2)$ & Sp (3) & $\mathrm{Ad}(4)$ & LS (5) & $\mathrm{Hb}(6)$ & & & & & \\
\hline Sample size & 145 & 145 & 145 & 145 & 145 & 145 & 145 & 145 & 145 & $p$ & 9 \\
\hline BL present & $77(53)$ & $81(56)$ & $87(60)$ & $93(64)$ & $91(62)$ & $88(60)$ & $99(69)$ & $108(75)$ & $114(78)$ & 0.136 & N \\
\hline BL absent & $23(16)$ & $22(15)$ & $21(15)$ & 19 (13) & $18(12)$ & $17(12)$ & $20(14)$ & $22(15)$ & $24(17)$ & 0.792 & $\Lambda$ \\
\hline $\mathrm{F}(+\mathrm{ve})$ & $36(25)$ & $31(21)$ & $19(13)$ & $9(6)$ & $6(4)$ & $8(6)$ & $15(10)$ & $6(4)$ & $5(3)$ & 0.056 & \\
\hline$F(-v e)$ & $3(2)$ & $4(3)$ & $5(3)$ & $7(5)$ & $8(6)$ & $9(6)$ & $6(4)$ & $4(3)$ & $1(1)$ & 0.057 & \\
\hline I/C & $6(4)$ & $7(5)$ & $13(9)$ & $17(12)$ & $22(16)$ & $23(16)$ & $5(3)$ & $5(3)$ & $1(1)$ & 0.0286 & $\mathrm{~N}$ \\
\hline AUC & $0.66 \pm 0.08$ & $0.69 \pm 0.05$ & $0.71 \pm 0.08$ & $0.73 \pm 0.04$ & $0.71 \pm 0.07$ & $0.68 \pm 0.06$ & $0.72 \pm 0.1$ & $0.76 \pm 0.11$ & $0.89 \pm 0.14$ & $<0.0001$ & 9.3 \\
\hline Sensitivity & $0.91 \pm 0.16$ & $0.96 \pm 0.06$ & $0.917 \pm 0.15$ & $0.88 \pm 0.08$ & $0.85 \pm 0.08$ & $0.68 \pm 0.07$ & $0.97 \pm 0.15$ & $0.972 \pm 0.18$ & 1 & 0.11 & N/ \\
\hline Accuracy & $0.72 \pm 0.15$ & $0.75 \pm 0.07$ & $0.78 \pm 0.05$ & $0.811 \pm 0.18$ & $0.79 \pm 0.09$ & $0.76 \pm 0.07$ & $0.86 \pm 0.14$ & $0.942 \pm 0.17$ & 1 & $<0.0001$ & 3.8 \\
\hline
\end{tabular}

Constant data are represented as number (percentage)

Surgery curative was considered as 'gold standard'

Chi-square independence test for constant data and One-way repeated-measures ANOVA following Dunnett Multiple comparisons tests for continuous data were used for statistical analysis

A $p$-value $<0.01$ was considered significant

A $q$-value $>4.148$ was considered significant

N/A Not applicable, BL Accurate the Bankart and bony Bankart lesions, I/C Inconclusive results, AUC Area under the curve, Ap Apprehension test, Re Relocation test, $S p$ Surprise test, Ad Anterior drawer test, $L S$ Load and shift test, $H b$ Hyperabduction test, $T R I$ The traditional radiological images, CET Clinical Examination Tests, $C E T+T R I$ Clinical Examination Tests combining the traditional radiological images, CT The Computed Tomography, SA Statistical analysis

$\mathrm{F}$ (+ve): False positive: The Bankart and/or bony Bankart lesions had found in diagnosis, which was led to surgery and actually it was the other bony problem $\mathrm{F}(-\mathrm{ve})$ : False negative: A bony problem had found in diagnosis, which was lead arthroscopy or open surgical procedure and actually it was the Bankart and/or bony Bankart lesions

have limited information to guide the surgeons in the decision making of surgeries for repair of the bony problem(s) of the shoulder [15]. In respect to the decisions of surgeons during the study, clinical examination tests combing traditional radiological images are failed in finding limitations of $\mathrm{CT}$ for quantification of the Bankart and bony Bankart lesions.

In the study, CT was provided the best visualization and clinical examination tests combing traditional radiological images were provided insufficient details of the Bankart and bony Bankart lesions for decision making of surgeries. This was because clinical examination tests combing traditional radiological images are failed to provide exact loss of the inferior glenoid [27]. These results were in line with available diagnostic studies [3, 28]. A retrospective study [29], literature reviews [4, 5, 25,27 , and a simulated study [30] are also concluded the same. But the published study is on fewer subjects (sample size: 70) [3] and more numbers of observers (four observers) [3, 28], which have less intra-observer and inter-observer reliabilities [22]. However, the results were not in line with the quantitative assessment of radiography and CT [30]. In respect to the results of the twin study, CT assessments have good agreements for decision making of surgeries of the Bankart and bony Bankart lesions.

Table 6 Performance parameters results of the adopted diagnostic methods for evaluation of the Bankart and bony Bankart lesions

\begin{tabular}{lll}
\hline Diagnostic modalities & $\begin{array}{l}\text { True positive the Bankart and bony Bankart } \\
\text { lesions ratio }\end{array}$ & $\begin{array}{l}\text { False positive the Bankart and bony Bankart } \\
\text { lesions ratio }\end{array}$ \\
\hline Computed Tomography & 0.79 & 0.03 \\
Traditional radiology & 0.68 & 0.10 \\
Without diagnosis images & 0.6 & 0.2 \\
Apprehension test & 0.53 & 0.25 \\
Relocation test & 0.56 & 0.21 \\
Surprise test & 0.6 & 0.13 \\
Anterior drawer test & 0.64 & 0.06 \\
Load and shift test & 0.63 & 0.04 \\
Hyperabduction test & 0.61 & 0.06 \\
Clinical Examination Tests combining the traditional & 0.75 & 0.15 \\
radiological images & & \\
\hline
\end{tabular}


Table 7 Beneficial score analysis of the adopted diagnostic methods for evaluation of the Bankart and bony Bankart lesions

\begin{tabular}{|c|c|c|c|c|c|c|c|c|c|c|c|}
\hline \multirow{3}{*}{$\mathrm{CP}$} & \multirow{3}{*}{ WF } & \multicolumn{9}{|c|}{ Decision making of the Bankart and bony Bankart lesions } & \\
\hline & & \multirow[t]{2}{*}{$\mathrm{CT}$} & \multirow[t]{2}{*}{$\mathrm{TRI}$} & \multirow[t]{2}{*}{ WDC } & \multicolumn{7}{|l|}{ CET } \\
\hline & & & & & $\mathrm{Ap}$ & $\operatorname{Re}$ & Sp & $\mathrm{Ad}$ & LS & $\mathrm{Hb}$ & CET + TRI \\
\hline 0 & 0 & 0.79 & 0.68 & 0.6 & 0.53 & 0.56 & 0.6 & 0.64 & 0.63 & 0.61 & 0.75 \\
\hline 0.1 & 0.11 & 0.78 & 0.67 & 0.58 & 0.50 & 0.53 & 0.59 & 0.63 & 0.62 & 0.60 & 0.73 \\
\hline 0.2 & 0.25 & 0.78 & 0.66 & 0.55 & 0.47 & 0.51 & 0.57 & 0.63 & 0.62 & 0.59 & 0.71 \\
\hline 0.3 & 0.43 & 0.77 & 0.64 & 0.51 & 0.43 & 0.47 & 0.54 & 0.61 & 0.61 & 0.58 & 0.68 \\
\hline 0.4 & 0.67 & 0.76 & 0.61 & 0.47 & 0.37 & 0.42 & 0.51 & 0.60 & 0.60 & 0.57 & 0.64 \\
\hline 0.5 & 1 & 0.75 & 0.58 & 0.4 & 0.28 & 0.35 & 0.47 & 0.60 & 0.59 & 0.55 & 0.59 \\
\hline 0.6 & 1.5 & 0.74 & 0.53 & 0.3 & 0.16 & 0.24 & 0.40 & 0.55 & 0.57 & 0.52 & 0.51 \\
\hline 0.7 & 2.33 & 0.71 & 0.44 & 0.13 & -0.05 & 0.06 & 0.29 & 0.50 & 0.53 & 0.48 & 0.38 \\
\hline 0.8 & 4 & 0.65 & 0.27 & -0.2 & -0.46 & -0.3 & 0.08 & 0.39 & 0.46 & 0.39 & 0.12 \\
\hline 0.9 & 9 & 0.48 & -0.24 & -1.2 & -1.70 & -1.37 & -0.58 & 0.082 & 0.26 & 0.11 & -0.67 \\
\hline 0.99 & 99 & -2.58 & -9.51 & -19.2 & -24.05 & -20.61 & -12.37 & -5.50 & -3.47 & -4.86 & -14.82 \\
\hline
\end{tabular}

CP A level of diagnostic confidence above its arthroscopy or opens surgical procedure had performed, WF Weighting factor (risk of overdiagnosis), CT The Computed Tomography, TRI The traditional radiological images, Ap Apprehension test, Re Relocation test, Sp Surprise test, Ad Anterior drawer test, LS Load and shift test, $H b$ Hyperabduction test, CET Clinical examination tests, CET + TRI Clinical examination tests combining the traditional radiological images, WDC Without diagnosis images or Clinical Examination Tests

Weighting factor $=\frac{\mathrm{A} \text { level of diagnostic confidence above its arthroscopy or opens surgical procedure had performed }}{1-\mathrm{A} \text { level of diagnostic confidence above its arthroscopy or opens surgical procedure had performed }}$

Decision making of the Bankart and bony Bankart lesions $=$ True positive ratio - (weighting factor $\times$ false positive ratio)

CT method was the increased financial burden of the patients. The traditional radiological images and clinical examination tests are cheaper than CT for diagnosis of the Bankart and bony Bankart lesions. However, CT has also prevented the future recurrence and/or failure of surgeries by exact diagnosis
[3]. Further research is required to justify the selection of CT for the diagnosis of the Bankart and bony Bankart lesions.

In the limitations of the study, for examples, the study was limited to diagnose the Bankart and bony Bankart lesions only no other type of shoulder

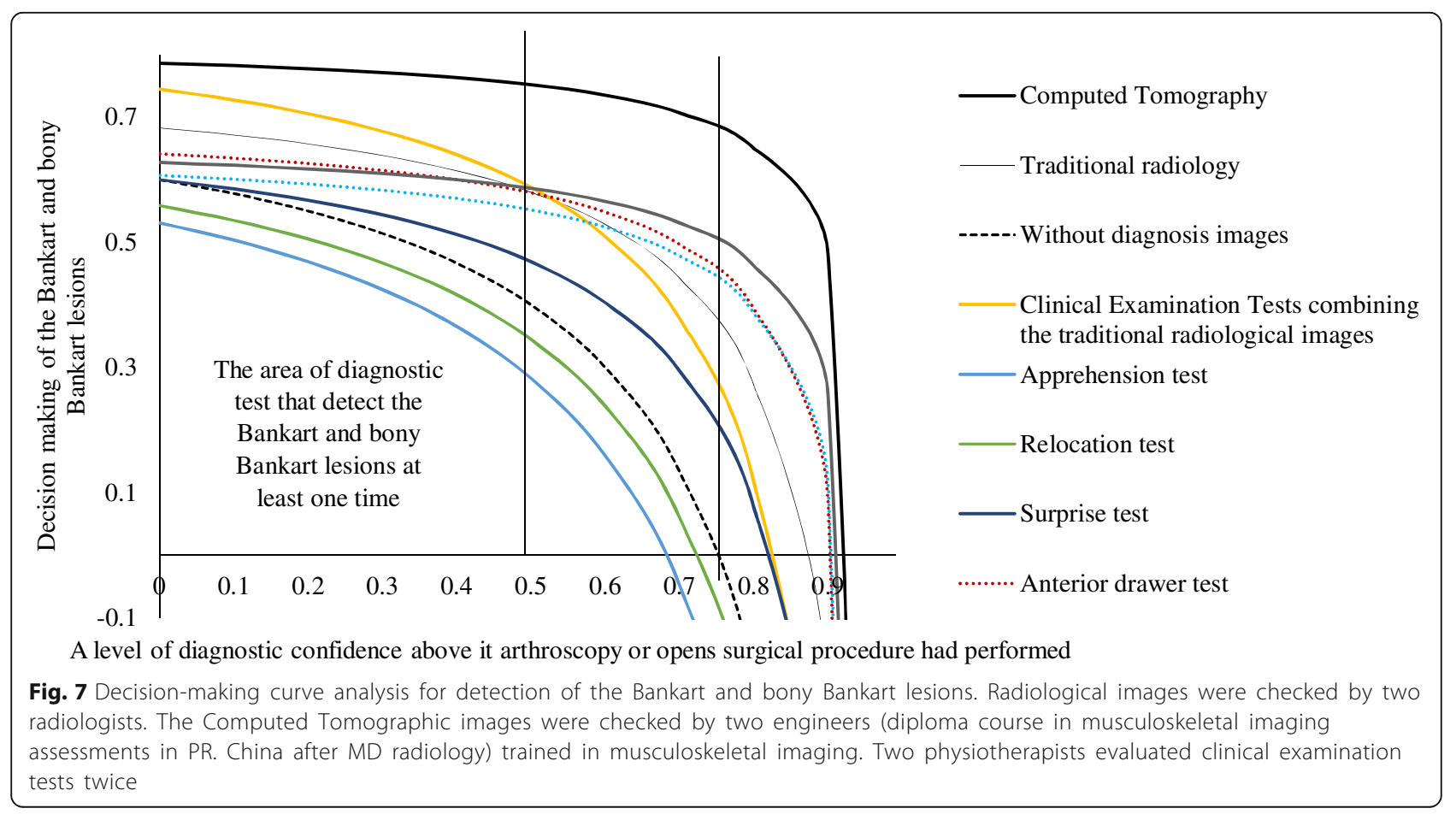




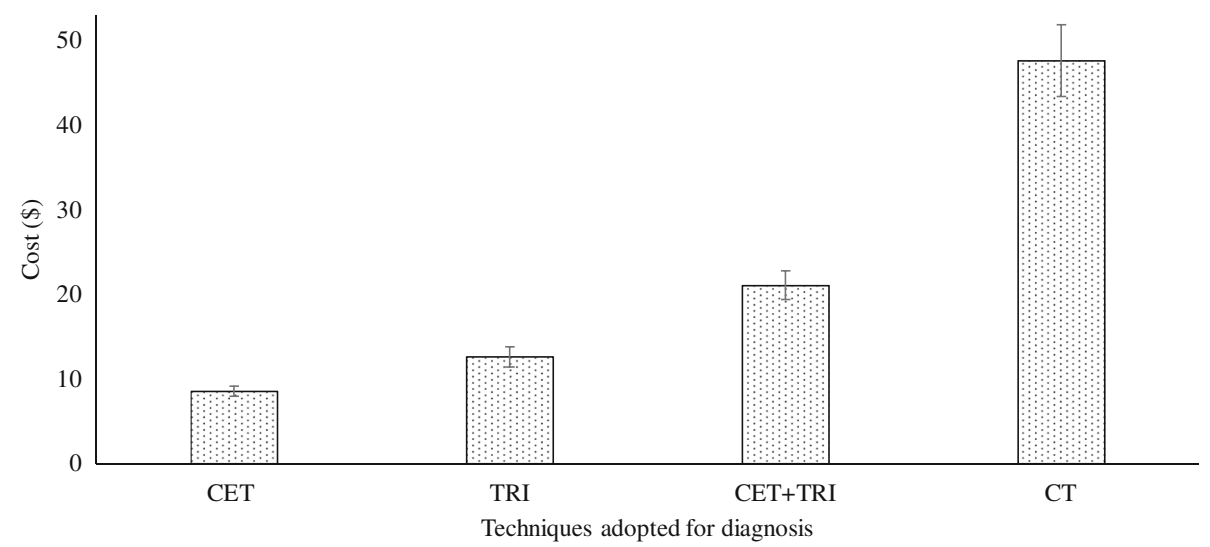

Fig. 8 Costs of techniques for the diagnosis of the Bankart and bony Bankart lesions. CET: Clinical examination tests, TRI: The traditional radiological images, CET + TRI: Clinical examination tests combining the traditional radiological images, CT: The Computed Tomography. Data were represented as mean \pm SD. Patients enrolled in the study (sample size: n): 145. One-way ANOVA following the Dunnett Multiple comparisons tests was used for statistical analysis. A $p$-value $<0.01$ and $q$-value $>4.148$ were considered significant

delocalization was addressed in the study. The study was not differentiated the Bankart and bony Bankart lesions. The evaluators of clinical examination tests were not blind to the demographic parameters of the enrolled patients. Imaging modalities like CT arthrography, MRI, MRI arthrography provide better soft tissue details compare to CT alone. The lack of 'gold standard' or further MRI. The possible justification is that further MRI only for head-to-head comparisons of diagnostic methods is vague in clinical practice. Therefore, researchers have not performed Further MRI.

\section{Conclusion}

The study concluded that the Computed Tomography has better performance parameters than clinical examination tests combining the traditional radiological images and should be considered for evaluation in patients with shoulder instability and the suspected Bankart and bony Bankart lesions.

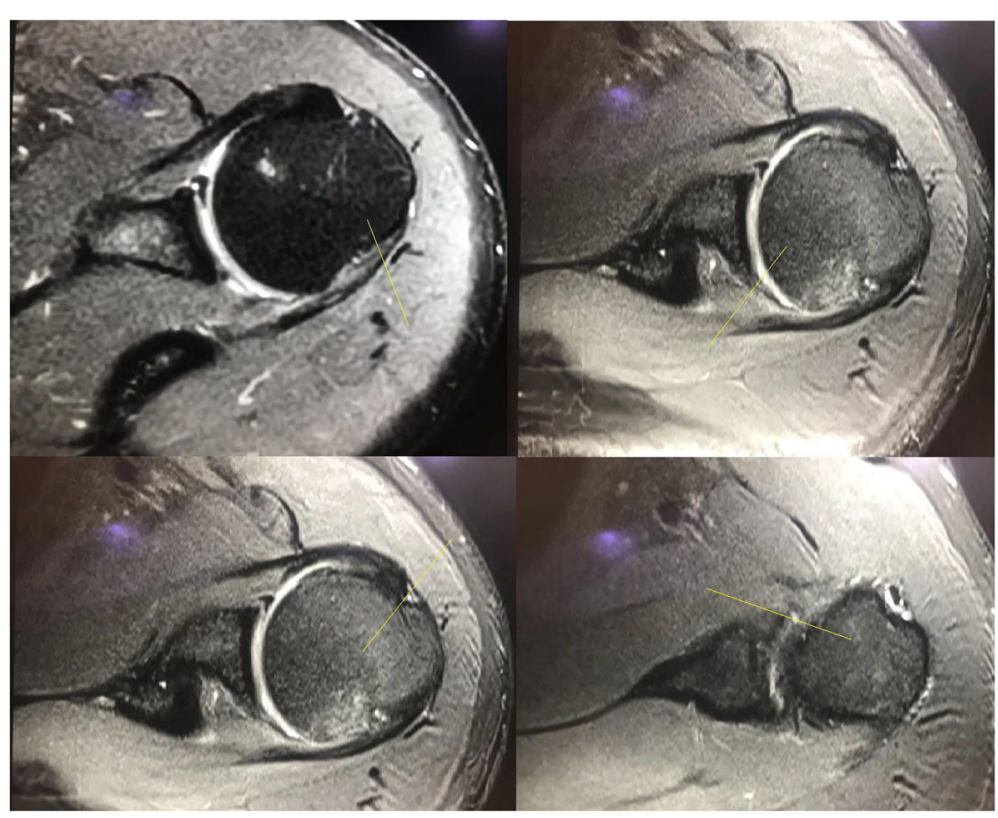

Fig. 9 Postsurgical radiographic images of 29-years female patient with shoulder instability showed the union of the grafts during follow-up. Supine view. The yellow line indicates the union of the Bankart lesions 


\section{Abbreviations}

ANOVA: Analysis of variance; CT: Computed Tomography; k: kappa value; MRI: Magnetic resonance imaging; STARD: Standards for reporting of diagnostic accuracy studies; STROCSS: The strengthening the reporting of cohort studies in surgery

\section{Acknowledgments}

Authors are like to thank for radiological, anesthetic, surgical, medical, and physiotherapist staff of PLA General Hospital, Beijing, China, Affiliated Hospital of Nantong University, Nantong Shi, China, and Dingbian County People's Hospital, Dingbian, China.

\section{Funding}

The research did not receive any specific financial motivation from government, non-government, or non-profitable sector to perform research.

\section{Availability of data and materials}

All information regarding patients were available from patients' Digital Imaging and Communications in Medicine files of Affiliated Hospital of Nantong University, China, Dingbian County People's Hospital, China, and PLA General Hospital, China.

\section{Authors' contributions}

All authors had read and approved a submission for publication. TL and JM contribute equally to conceptualization and methodology of the study. HC contributed to methodology, data curation, and formal analysis of the study. $\mathrm{DH}$ contributed to investigation, resources, and software of the study. LX was project administrator, contributed to data curation, the manuscript preparation, the manuscript editing, and the manuscript review for the intellectual content. The author agrees to be accountable for all aspects of work ensuring integrity and accuracy.

\section{Ethics approval and consent to participate}

The study had been registered in the research registry (www.researchregistry.com), UID No.: researchregistry3990 dated 15 December 2014. The protocol (DI/HR/27/14, dated 15 November 2014) had been approved by the PLA General Hospital review board. The enrolled patients signed an informed consent form regarding radiology and surgeries.

\section{Consent for publication}

The enrolled patients signed an informed consent form regarding the publication of the study including patients' personal images (if any) in all formats (hard and/or electronics) irrespective of time and language.

\section{Competing interests}

Authors have reported that they have no commercial or associated interest that represents a conflict of interest in connection with results and/or discussion reported the submitted manuscript.

\section{Publisher's Note}

Springer Nature remains neutral with regard to jurisdictional claims in published maps and institutional affiliations.

\section{Author details}

'Department of Medical Imaging, Affiliated Hospital of Nantong University, Shi, Jiangsu Sheng, Nantong 226001, China. ${ }^{2}$ Department of Magnetic Resonance Imaging, Dingbian County People's Hospital, Dingbian, Yulin 718600, Shaanxi, China. ${ }^{3}$ Department of Radiology, PLA general hospital, No.28 Fuxing Road, Haidian District, Beijing 100000, China.

\section{Received: 23 August 2018 Accepted: 9 November 2018}

\section{Published online: 23 November 2018}

\section{References}

1. Antunes JP, Mendes A, Prado MH, Moro OP, Miro RL. Arthroscopic Bankart repair for recurrent shoulder instability: a retrospective study of 86 cases. J Orthop. 2016;13:95-9.

2. Auffarth A, Mayer M, Kofler B, Hitzl W, Bogner R, Moroder P, Korn G, Koller $H$, Resch $H$. The interobserver reliability in diagnosing osseous lesions after first-time anterior shoulder dislocation comparing plain radiographs with computed tomography scans. J Shoulder Elb Surg. 2013;22:1507-13.
3. Delage RA, Balg F, Bouliane MJ, Canet-Silvestri F, Garant-Saine L, Sheps DM, Lapner P, Rouleau DM. Indication for computed tomography scan in shoulder instability: sensitivity and specificity of standard radiographs to predict bone defects after traumatic anterior glenohumeral instability. Orthop J Sports Med. 2017;5. https://doi.org/10.1177/2325967117733660.

4. Auffarth $\mathrm{A}$, Matis $\mathrm{N}$, Koller $\mathrm{H}$, Resch $\mathrm{H}$. An alternative technique for the exact sizing of glenoid bone defects. Clin Imaging. 2012;36:574-6.

5. Skupinski J, Piechota MZ, Wawrzynek W, Maczuch J, Babinska A. The bony Bankart lesion: how to measure the glenoid bone loss. Pol J Radiol. 2017;82: 58-63.

6. Weel $H$, Tromp W, Krekel PR, Randelli P, van den Bekerom MP, van Deurzen DF. International survey and surgeon's preferences in diagnostic work-up towards treatment of anterior shoulder instability. Arch Orthop Trauma Surg. 2016;136:741-6.

7. Ho AG, Gowda AL, Michael WJ. Evaluation and treatment of failed shoulder instability procedures. J Orthopaed Traumatol. 2016;17:187-97.

8. Wang L, Liu Y, Su X, Liu S. A meta-analysis of arthroscopic versus open repair for treatment of bankart lesions in the shoulder. Med Sci Monit. 2015, 21:3028-35.

9. Bois AJ, Fening SD, Polster J, Jones MH, Miniaci A. Quantifying glenoid bone loss in anterior shoulder instability: reliability and accuracy of 2-dimensional and 3-dimensional computed tomography measurement techniques. Am J Sports Med. 2012;40:2569-77.

10. Provencher MT, Bhatia S, Ghodadra NS, Grumet RC, Bach BR Jr, Dewing CB, LeClere L, Romeo AA. Recurrent shoulder instability: current concepts for evaluation and management of glenoid bone loss. J Bone Joint Surg Am. 2010;92:133-51.

11. Hendry JH, Simon SL, Wojcik A, Sohrabi M, Burkart W, Cardis E, Laurier D, Tirmarche M, Hayata I. Human exposure to high natural background radiation: what can it teach us about radiation risks? J Radiol Prot. 2009;29: A29-42.

12. Agha RA, Borrelli MR, Vella-Baldacchino M, Thavayogan R, Orgill DP, STROCSS Group. The STROCSS statement: strengthening the reporting of cohort studies in surgery. Int J Surg. 2017:46:198-202.

13. van Kampen DA, van den Berg T, van der Woude HJ, Castelein RM, Terwee CB, Willems WJ. Diagnostic value of patient characteristics, history, and six clinical tests for traumatic anterior shoulder instability. J Shoulder Elb Surg. 2013:22:1310-9.

14. van der Linde JA, van Kampen DA, van Beers LW, van Deurzen DF, Terwee CB, Willems WJ. The Oxford shoulder instability score; validation in Dutch and first-time assessment of its smallest detectable change. J Orthop Surg Res. 2015;10. https://doi.org/10.1186/s13018-015-0286-5.

15. Kumar K, Makandura M, Leong NJ, Gartner L, Lee CH, Ng DZ, Tan CH, Kumar VP. Is the apprehension test sufficient for the diagnosis of anterior shoulder instability in young patients without magnetic resonance imaging (MRI)? Ann Acad Med Singap. 2015;44:178-84.

16. Ebrahimzadeh $\mathrm{MH}$, Moradi A, Zarei AR. Minimally invasive modified latarjet procedure in patients with traumatic anterior shoulder instability. Asian J Sports Med. 2015;6. https://doi.org/10.5812/asjsm.26838.

17. Lizzio VA, Meta F, Fidai M, Makhni EC. Clinical evaluation and physical exam findings in patients with anterior shoulder instability. Curr Rev Musculoskelet Med. 2017;10:434-41.

18. Park JY, Kim Y, Oh KS, Lim HK, Kim JY. Stress radiography for clinical evaluation of anterior shoulder instability. J Shoulder Elb Surg. 2016;25: e339-47.

19. Morita W, Tasaki A. Intra- and inter-observer reproducibility of shoulder laxity tests: comparison of the drawer, modified drawer and load, and shift tests. J Orthop Sci. 2018;23:57-63.

20. Barchilon VS, Kotz E, Barchilon Ben-Av M, Glazer E, Nyska M. A simple method for quantitative evaluation of the missing area of the anterior glenoid in anterior instability of the glenohumeral joint. Skelet Radiol. 2008;37:731-6.

21. Fitzgerald M, Saville BR, Lewis RJ. Decision curve analysis. JAMA. 2015;13: 409-10

22. Dixon SN, Donner A, Shoukri MM. Adjusted inference procedures for the interobserver agreement in twin studies. Stat Methods Med Res. 2016;25: 1260-71.

23. Thomazeau H, Courage O, Barth J, Veillard D, Boileau P, Society FA. Can we improve the indication for Bankart arthroscopic repair? A preliminary clinical study using the ISIS score. Orthop Traumatol Surg Res. 2010;96:S77-88.

24. Bushnell BD, Creighton RA, Herring MM. Bony instability of the shoulder. Arthroscopy. 2008;24:1061-73. 
25. Arciero RA, Parrino A, Bernhardson AS, Diaz-Doran V, Obopilwe E, Cote MP, Golijanin P, Mazzocca AD, Provencher MT. The effect of a combined glenoid and hill-Sachs defect on glenohumeral stability: a biomechanical cadaveric study using 3-dimensional modeling of 142 patients. Am J Sports Med. 2015;43:1422-9.

26. Hanchard NC, Lenza M, Handoll HH, Takwoingi Y. Physical tests for shoulder impingements and local lesions of bursa, tendon or labrum that may accompany impingement. Cochrane Database Syst Rev. 2013. https://doi. org/10.1002/14651858.CD00742.

27. Sugaya $\mathrm{H}$. Techniques to evaluate glenoid bone loss. Curr Rev Musculoskelet Med. 2014;7:1-5.

28. Milano G, Saccomanno MF, Magarelli N, Bonomo L. Analysis of agreement between computed tomography measurements of glenoid bone defects in anterior shoulder instability with and without comparison with the contralateral shoulder. Am J Sports Med. 2015:43:2918-26.

29. Loh B, Lim JB, Tan AH. Is clinical evaluation alone sufficient for the diagnosis of a Bankart lesion without the use of magnetic resonance imaging? Ann Transl Med. 2016;4. https://doi.org/10.21037/atm.2016.11.22.

30. Itoi E, Lee SB, Amrami KK, Wenger DE, An KN. Quantitative assessment of classic anteroinferior bony Bankart lesions by radiography and computed tomography. Am J Sports Med. 2013;31:112-1128.

Ready to submit your research? Choose BMC and benefit from:

- fast, convenient online submission

- thorough peer review by experienced researchers in your field

- rapid publication on acceptance

- support for research data, including large and complex data types

- gold Open Access which fosters wider collaboration and increased citations

- maximum visibility for your research: over $100 \mathrm{M}$ website views per year

At $\mathrm{BMC}$, research is always in progress.

Learn more biomedcentral.com/submissions 